\title{
Which peer-reviewed journals publish the most on diabetes?'
}

\author{
Christine J. Neilson and Daniel D. Neilson
}

\begin{abstract}
Objectives - A list of the major peer-reviewed journals that publish on diabetes has not been published to date. This article briefly describes a project to compile a list of peer-reviewed English-language journals that publish the most on the topic of diabetes. Methods - We used the PubMed and CINAHL Plus databases to identify relevant articles published in English between 2003 and 2007. The number of citations for each journal was totalled, and the peer-reviewed status of the journals topping the list was determined. Results - We present a list of 100 peer-reviewed journals relevant to the topic of diabetes that is based on a larger list compiled for the Canadian Diabetes Educator Certification Board. The list includes a mix of general medicine journals and specialty journals. Discussion - A number of technical setbacks were overcome to determine which scholarly journals publish the most articles of potential interest to health practitioners with an interest in diabetes. This list can inform the current awareness activities of librarians and health practitioners alike, and may be useful from the perspective of collection development.
\end{abstract}

\section{Objectives}

Since 2005, diabetes educators in Canada have had the option of maintaining their certification through a credit portfolio that documents participation in continuing education activities, including reading relevant peer-reviewed journal articles [1]. There are several journals devoted to diabetes, but diabetes is a complex condition that affects various body systems, so relevant articles could potentially be found in almost any health-related journal. To complicate matters further, it is not always obvious whether or not a journal is peer-reviewed.

A diabetes educator involved in administering the Canadian Diabetes Educator Certification Board's portfolio program approached one of the authors to obtain a list of the major peer-reviewed diabetes-related journals to help the program auditors determine which journal articles are acceptable for credit. A literature search and Web search for such a list did not return any relevant results, and a posting on the CANMED-lib listserv only resulted in responses from individuals who wanted a copy of the list as well once one was located. To fill this information gap, we compiled a list of peer-reviewed journals relevant to the subject of diabetes.

\section{Methods}

We searched the PubMed and CINAHL Plus databases for relevant articles that were published in English between
2003 and 2007, using a combination of subject headings and keywords. The search terms used for PubMed were "Diabetes Mellitus" [Mesh] OR diabet* OR "Hemoglobin A, Glycosylated" [Mesh] OR a1c OR "Hyperglycemia" [Mesh] OR "Hypoglycemia" [Mesh] OR "glycemic control" OR "Diabetes Complications" [Mesh] OR "diabetes education" OR "Insulin" [Mesh]. The search terms used for CINAHL Plus were (MH "Diabetes Mellitus+") OR diabet* OR (MH "Hemoglobin A, Glycosylated") OR a1c OR (MH "Hyperglycemia+") OR (MH "Hypoglycemia+") OR (MH "Glycemic Control") OR "diabetes complications" OR (MH "Diabetes Education") OR (MH "Insulin+"). Exploded subject headings were used where available to ensure the results were as comprehensive as possible. We also chose to use the truncated term diabet* to retrieve records that did not yet have subject headings assigned to them and to maximize the sensitivity of the search. All article types were included.

The resulting citations were imported into custom software developed specifically for this project to identify and remove duplicate records, and determine how many articles from the results were published in each year for each journal title. Data entry errors in and data inconsistencies between databases made the use of journal titles and journal ID numbers unreliable for detecting duplicate articles. We found that in many cases the recorded journal titles differed in punctuation, spelling, and/or abbreviation style. On the other hand, we found that article PMID numbers were generally

C.J. Neilson. ${ }^{2}$ Saskatchewan Health Information Resources Partnership, University of Saskatchewan, Health Sciences Library,

107 Wiggins Road, Saskatoon, SK S7N 5E5, Canada (e-mail: christine.neilson@usask.ca).

D.D. Neilson. Department of Computing Science, University of Alberta, Edmonton, AB T6G 2E8, Canada

(e-mail: dneilson@ualberta.net).

${ }^{1}$ This article has been peer-reviewed.

${ }^{2}$ Corresponding author. 
more reliable, so our software identified which journal ID numbers were equivalent by comparing the journal ID numbers from records with the same PMID. A manual check for equivalence was conducted for journal IDs that did not have records with a PMID. Once journal ID equivalence was determined, the software identified and removed duplicate records by comparing citation information. Specifically, the software used article title, author(s), journal ID, year of publication, volume, and issue number to identify duplicate records. Article title and author lists were compared by stripping them of white space (spaces, tabs, etc.) and changing them to all capital letters because data entry errors in the databases make them otherwise incomparable.

Breaking down the number of citations by year provided a visual cue to determine whether a journal had been discontinued or had undergone a title change. Where a title had changed during the time period examined, the number of citations for the old and new titles were added together.

The top 300 journal titles, by number of articles published, were checked for peer-review status using publication information in CINAHL Plus, publisher Web sites, and e-mail inquiries to individual publishers. In cases where a publisher did not respond to the authors' inquiries, the journal was labelled as "unknown" and was considered not to be peerreviewed for the purposes of the list presented in Appendix A.

Data analysis consisted of dividing the total number of citations retrieved for a given title by the total number of issues (including supplements) published during the 5-year time period. A list of the top 100 titles, as determined by the article/issue ratio, was created to compare with the top 100 titles based on the total number of citations retrieved for each title.

\section{Results}

A total of 100914 unique article citations from 4246 individual journal titles were retrieved from PubMed and CINAHL Plus for the 5-year period from 2003 to 2007. Out of the top 300 titles by number of articles published, 259 were peer-reviewed. Twenty-seven titles were not peerreviewed publications-including two which were directed at health consumers rather than health professionals-and the peer-reviewed status of 14 titles could not be determined (see Appendix B).

The top 100 peer-reviewed journal titles are listed in Appendix A. They include a mix of general medicine journals and specialty journals covering topics such as diabetes, endocrinology, nephrology, cardiology, chemistry, pharmacology, internal medicine, and nutrition.

\section{Discussion}

The obstacles encountered through the course of this project were largely technical in nature. The volume of citations involved was unwieldy for commercial citation management software and made export from CINAHL Plus particularly cumbersome. As a result, the development of custom software was required to overcome the limitations of the commercial software. Data entry errors and inconsistencies posed a challenge to programming our software, as discussed in the Methods above.
Technical issues aside, there are some limitations to the method used to create this list. First, the results were shaped by the databases used. These two databases were chosen because they are generally accessible to practitioners; PubMed is freely available via the Internet, and CINAHL Plus is available through many institutions, as well as the NurseONE portal for Canadian registered nurses [2]. PubMed's focus on clinical medicine and CINAHL Plus' focus on nursing and allied health offer a more varied set of titles indexed than if we had used only one database or the other. However, some relevant journals may not be indexed in either database, while other journals may only be partially indexed. Retrospective indexing may take place, which would change the citation pool, changing the list rankings over time even though the time frame examined remains the same.

Secondly, even though we attempted to conduct a comprehensive search, the search terms used may not have retrieved all relevant articles. Some newer articles may not yet have been assigned subject headings, and some relevant articles may not have been assigned the headings that we used. A search for diabet* offsets this somewhat, but PubMed cuts off the results of a truncated search after the first 600 citations, so some citations could have been missed.

Finally, journal quality was not taken into account when compiling the list. An argument can be made for ranking the titles in terms of quality rather than quantity, but given the original request was to provide a list of peer-reviewed titles that practitioners could count for credit (not a list of the "best" peer-reviewed journals), this was not done. However, after examining the list, we would argue that many of the journals listed are well known and are considered to be reputable publications.

Journals that are published weekly or even monthly have a potential advantage over those that are published less frequently in terms of the overall quantity of articles published and how high they appear on the list. While the concentration of articles for each journal was unimportant for the original purpose of the larger list created for the Canadian Diabetes Educator Certification Board (to provide a reference list of journals for determining whether an article is acceptable for continuing education credit), the concentration of relevant articles plays a larger role for the purposes of current awareness activities and collection development. Table A1 (see Appendix A) provides two versions of the top 100 list: the left column lists the journals sorted by the raw number of articles published on diabetes during the 5-year period and the right column lists the journals sorted by the number of articles divided by the number of issues (including supplements). The article/issue ratio is intended to give an indication of which journals have a higher concentration of articles relating to diabetes.

Most of the journals listed appear in both versions of the list even though they may occupy a different place in the order. However, there are some journals that appeared in one list but not the other. Notably, European Diabetes Nursing, Journal of the Cardiometabolic Syndrome, and Clinical Diabetes are included in the top 25 journals listed by the article/issue ratio, but these journals did not publish a large enough quantity of articles to be listed at all in the list sorted by quantity. Meanwhile, the prominent journals BMJ, JAMA, Lancet, and New England Journal of Medicine published 
enough relevant items to be listed in the top 100 journals listed by the raw number of articles but did not have a large enough article/issue ratio to be included in the list sorted by concentration of diabetes articles.

It is unsurprising that many of the journals at the top of the list are focused solely on diabetes. Given the method used to compile the list, one would likely question the list's validity if this had not been the case. However, one of the most interesting things about the two versions of the list is that the top six journals are the same for both. Diabetes Care, Diabetes, Diabetic Medicine, Diabetologica, Diabetes Research and Clinical Practice, and Journal of Clinical Endocrinology and Metabolism rose to the top in terms of both quantity and concentration of articles, the only change being that Diabetic Medicine and Diabetologica switched positions in the list.

Despite the limitations mentioned above, this list is useful for identifying peer-reviewed journals to monitor for
English-language articles relevant to diabetes and is informative for collection development purposes.

\section{References}

1. Canadian Diabetes Educators Certification Board (CDECB). How To Guide: Collecting certification maintenance credits [monograph on the Internet]. Inglewood, Ont.: CDECB; 2007 [cited 2008 March 1]. Available from http://www.cdecb.ca/ Docs/HowtoGuideEntire.pdf.

2. Canadian Nurses Association. NurseONE.ca Fact Sheets [fact sheet on the Internet]. Ottawa, Ont.: Canadian Nurses Association; 2008 [cited 2008 July 21]. Most recent version available from http://www.cna-nurses.ca/CNA/documents/pdf/ publications/2008_Fact_Sheets_e.pdf.

Appendix A begins on the following page. 


\section{Appendix A}

Table A1. Top 100 journal titles publishing on diabetes.

\begin{tabular}{|c|c|c|}
\hline Journals by no. of articles published & Rank & Journals by articles/issues ${ }^{a}$ \\
\hline Diabetes Care (3490) & 1 & Diabetes Care (52.09) \\
\hline Diabetes (2580) & 2 & Diabetes $(40.31)$ \\
\hline Diabetic Medicine (1550) & 3 & Diabetologia (20.91) \\
\hline Diabetologia (1359) & 4 & Diabetic Medicine (20.13) \\
\hline Diabetes Research and Clinical Practice (1235) & 5 & Diabetes Research and Clinical Practice (19.3) \\
\hline Journal of Clinical Endocrinology and Metabolism (1111) & 6 & $\begin{array}{l}\text { Journal of Clinical Endocrinology and Metabolism } \\
\text { (18.21) }\end{array}$ \\
\hline $\begin{array}{l}\text { American Journal of Physiology, Endocrinology and } \\
\text { Metabolism (743) }\end{array}$ & 8 & Diabetes Technology and Therapeutics (13.77) \\
\hline Circulation (630) & 9 & $\begin{array}{l}\text { American Journal of Physiology, Endocrinology and } \\
\text { Metabolism (12.38) }\end{array}$ \\
\hline Transplantation Proceedings (587) & 10 & Diabetes Management Journal (11.82) \\
\hline Diabetes Educator (567) & 11 & Diabetes Spectrum (11.35) \\
\hline American Journal of Clinical Nutrition (494) & 14 & Journal of Diabetes and its Complications (9.58) \\
\hline Kidney International (565) & 15 & European Diabetes Nursing (8.56) \\
\hline Diabetes Technology and Therapeutics (427) & 16 & Pediatric Diabetes (8.39) \\
\hline Nephrology, Dialysis, Transplantation (419) & 17 & Endocrinology (8.28) \\
\hline $\begin{array}{l}\text { Proceedings of the National Academy of Sciences of } \\
\text { the United States of America (408) }\end{array}$ & 18 & American Journal of Clinical Nutrition (8.23) \\
\hline $\begin{array}{l}\text { Obesity (Silver Spring, Md.), formerly Obesity Research } \\
\text { (379) }\end{array}$ & 19 & Cochrane Database of Systematic Reviews (8.05) \\
\hline Journal of the American Society of Nephrology (375) & 20 & Journal of the Cardiometabolic Syndrome (7.89) \\
\hline American Journal of Kidney Diseases (361) & 21 & Acta Diabetologica (7.46) \\
\hline Practical Diabetes International (359) & 22 & Practical Diabetes International (7.33) \\
\hline Atherosclerosis (341) & 23 & Endocrine Practice (6.95) \\
\hline JAMA (329) & 29 & Journal of the American Society of Nephrology (5.28) \\
\hline Journal of the American College of Cardiology (309) & 30 & Hormone and metabolic research $(5.17)$ \\
\hline European Journal of Endocrinology (308) & 31 & Kidney International (5.14) \\
\hline Hormone and Metabolic Research (304) & 32 & Review of Diabetic Studies (5.13) \\
\hline Clinical Endocrinology (302) & 33 & Clinical Endocrinology (5.03) \\
\hline Journal of Diabetes and its Complications (297) & 34 & Journal of Hypertension (4.99) \\
\hline American Heart Journal (291) & 35 & Endocrine Journal (4.93) \\
\hline Archives of Internal Medicine (281) & 36 & Vascular Health and Risk Management (4.93) \\
\hline Endocrine Practice (278) & 37 & European Journal of Endocrinology (4.53) \\
\hline Pediatric Diabetes (277) & 38 & Preventing Chronic Disease (4.41) \\
\hline Lancet (272) & 39 & $\begin{array}{l}\text { International Journal of Obesity (2005), formerly Interna- } \\
\text { tional Journal of Obesity and Related Metabolic } \\
\text { Disorders (4.38) }\end{array}$ \\
\hline BMJ (269) & 40 & Nephrology, Dialysis, Transplantation (4.32) \\
\hline Journal of Clinical Investigation (266) & 41 & Stroke $(4.31)$ \\
\hline Stroke $(263)$ & 42 & American Heart Journal (4.28) \\
\hline Journal of Immunology (Baltimore, Md., 1950) (244) & 43 & American Journal of Kidney Diseases (4.10) \\
\hline
\end{tabular}


Table A1. (continued).

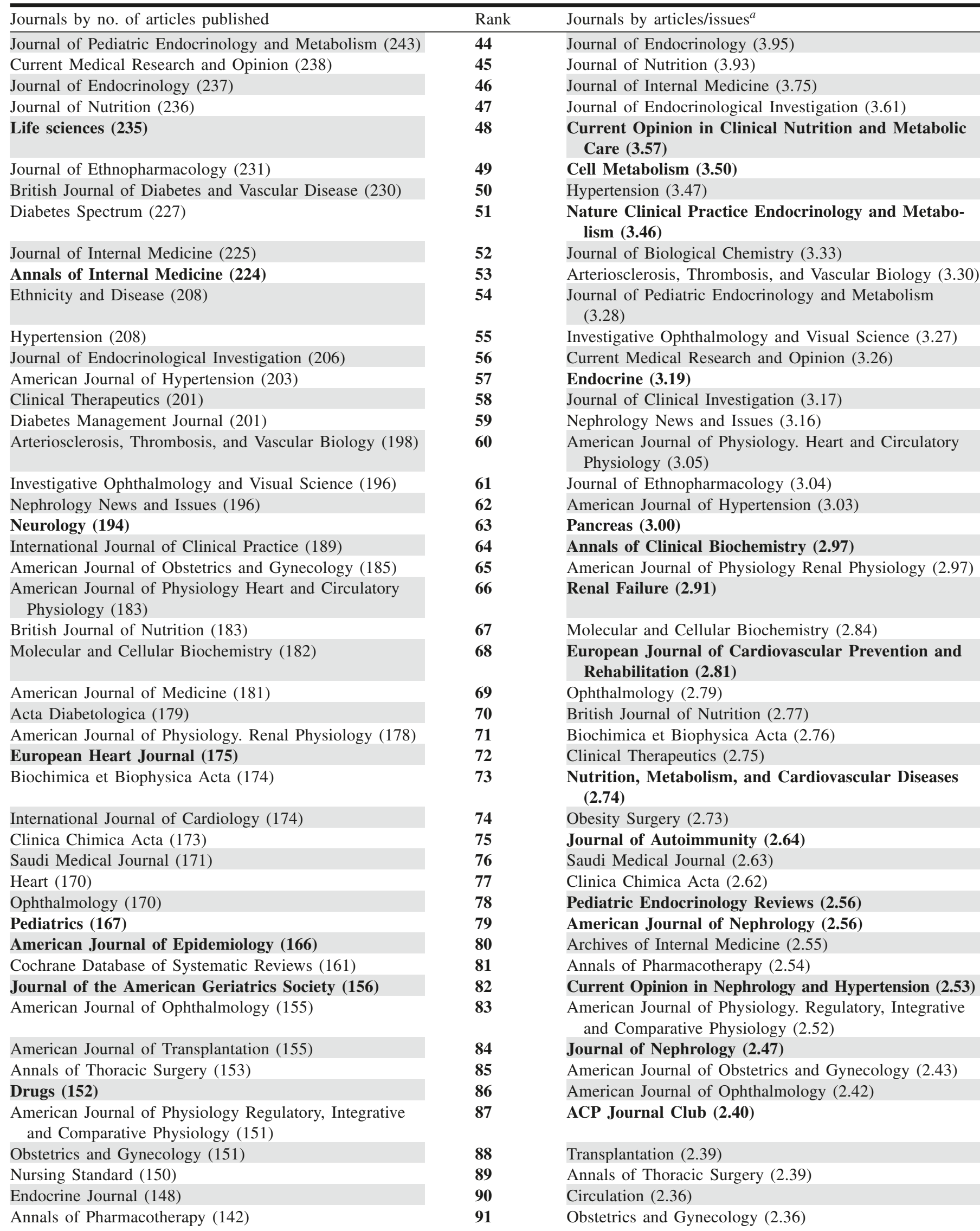


Table A1. (concluded).

\begin{tabular}{lll}
\hline Journals by no. of articles published & Rank & Journals by articles/issues $^{a}$ \\
\hline FEBS Letters (142) & $\mathbf{9 2}$ & International Journal of Clinical Practice (2.33) \\
Hormone Research (140) & $\mathbf{9 3}$ & Nutrition (2.29) \\
American Family Physician (139) & $\mathbf{9 4}$ & Journal of Atherosclerosis and Thrombosis (2.23) \\
Biochemical Journal (138) & $\mathbf{9 5}$ & Heart (2.21) \\
Advanced Studies in Medicine (137) & $\mathbf{9 6}$ & Growth Hormone and IGF Research (2.18) \\
Blood (136) & $\mathbf{9 7}$ & Archives of Medical Research (2.18) \\
European Journal of Clinical Investigation (135) & $\mathbf{9 8}$ & Journal of Pharmacology and Experimental Therapeu- \\
& & tics (2.17) \\
Obesity Surgery (134) & $\mathbf{9 9}$ & Molecular Endocrinology (Baltimore, Md.) (2.15) \\
Journal of the Association of Physicians of India (133) & $\mathbf{1 0 0}$ & American Journal of Transplantation (2.12) \\
\hline
\end{tabular}

Note: Journals that are not included on both sides of the list are in bold.

${ }^{a}$ Includes supplements.

\section{Appendix B}

Table B1. Non-peer-reviewed titles from the original top 300 list.

Not peer-reviewed
Annals of the New York Academy of Sciences
AHRQ Research Activities
Biochemical Society transactions
Clinical Advisor for Nurse Practitioners
Current Atherosclerosis Reports
Current Diabetes Reports
Current Hypertension Reports
Current Medicinal Chemistry
Diabetes Forecast (consumer audience)
Diabetes Self-Management (consumer audience)
Diabetes/Metabolism Research and Reviews
Diabetic Foot
Endocrinology and Metabolism Clinics of North America
Foot and Ankle International
International Journal of Lower Extremity Wounds
Iranian Journal of Diabetes and Lipid Disorders
Journal of Diabetes Nursing
Medical Hypotheses
MMWR: Morbidity and Mortality Weekly Report
Nursing Times
Ocular Surgery News
Patient Care
Podiatry Management
Practice Nurse
Proceedings of the Nutrition Society
Reviews in Endocrine and Metabolic Disorders
Wounds: A Compendium of Clinical Research and Practice

Peer-reviewed status unknown

Advances in Experimental Medicine and Biology

Asia Pacific Journal of Clinical Nutrition

Biological and Pharmaceutical Bulletin

Clinical chemistry

Annual International Conference of the IEEE Engineering in Medicine and Biology Society - IEEE Engineering in Medicine and Biology Society Conference Proceedings.

Current Opinion in Lipidology

Diabetes and Metabolism

Diabetes and Vascular Disease Research

Diabetes, Nutrition and Metabolism

European Journal of Pharmacology

Experimental and Clinical Endocrinology and Diabetes

Metabolism: Clinical and Experimental

Molecular Genetics and Metabolism

Treatments in Endocrinology 